\title{
Prospective study assessing three-dimensional changes of mucosal healing following soft tissue augmentation using free gingival grafts
}

\author{
Puria Parvini $^{1} \quad$ | Maria Elisa Galarraga-Vinueza ${ }^{1,2,5} \quad$ | Karina Obreja $^{1}$ | \\ Ricardo de Sousa Magini $^{2}$ ｜ Robert Sader $^{3} \quad$ | Frank Schwarz ${ }^{1,4}$ \\ ${ }^{1}$ Department of Oral Surgery and Implantology, Johann Wolfgang Goethe-University Frankfurt, Carolinum, Frankfurt, Germany \\ ${ }^{2}$ Post-Graduate Program in Implant Dentistry (PPGO), Federal University of Santa Catarina (UFSC), Florianópolis, SC, Brazil \\ ${ }^{3}$ Department for Oral, Cranio-Maxillofacial and Facial Plastic Surgery, Medical Center of the Goethe University Frankfurt, Frankfurt am Main, \\ Germany \\ ${ }^{4}$ Department of Oral Surgery, Universitätsklinikum Düsseldorf, Düsseldorf, Germany \\ ${ }^{5}$ School of Dentistry, Universidad de las Américas, Quito, Ecuador
}

\section{Correspondence}

Maria Elisa Galarraga-Vinueza, Department of Oral Surgery and Implantology, Carolinum, Goethe University, TheodorStern-Kai 7, 60596 Frankfurt am Main, Germany.

Email:m.galarraga@med.uni-frankfurt.de

[Correction added on 18 September 2020, after first online publication: Projekt Deal funding statement has been added.]

Funding information Osteology Foundation, LucerneSwitzerland

\begin{abstract}
Background: The present study aimed to assess the three-dimensional changes following soft tissue augmentation using free gingival grafts (FGG) at implant sites over a 3-month follow-up period.

Methods: This study included 12 patients exhibiting deficient keratinized tissue (KT) width (i.e., $<2 \mathrm{~mm}$ ) at the vestibular aspect of 19 implants who underwent soft tissue augmentation using FGG at second stage surgery following implant placement. Twelve implants were considered for the statistical analysis $(n=12)$. The region of interest (ROI) was intraorally scanned before surgery (S0), immediately post-surgery (S1), 30 (S2) and 90 (S3) days after augmentation. Digital scanned files were used for quantification of FGG surface area (SA) and converted to standard tessellation language (STL) format for superimposition and evaluation of thickness changes between the corresponding time points. FGG shrinkage (\%) in terms of SA and thickness was calculated between the assessed time points.

Results: Mean FGG SA amounted to 91 (95\% CI: 63 to 119), 76.2 (95\% CI: 45 to 106), and 61.3 (95\% CI: 41 to 81) $\mathrm{mm}^{2}$ at S1, S2, and S3, respectively. Mean FGG SA shrinkage rate was $16.3 \%$ (95\% CI: 3 to 29 ) from S1 to S2 and 33\% (95\% CI: 19 to 46 ) from S1 to S3. Mean thickness gain from baseline (S0) to S1, S2, and S3 was 1.31 (95\% CI: 1.2 to 1.4 ), 0.82 (95\% CI: 0.5 to 1.12 ), and 0.37 (0.21 to 0.5 ) $\mathrm{mm}$, respectively. FGG thickness shrinkage was of 38\% (95\% CI: 17.6 to 58) from S1 to S2 and 71.8\% (95\% CI: 60 to 84) from S1 to S3. Dimensional changes from S1 to S3 were statistically significant, $P<0.017$. Soft tissue healing was uneventful in all patients.
\end{abstract}


Conclusions: The present three-dimensional assessment suggests that FGG undergo significant dimensional changes in SA and thickness over a 3-month healing period.

KEYWORDS

dental implants, free gingival graft, keratinized mucosa, shrinkage, three-dimensional analysis

\section{1 | INTRODUCTION}

The relevance of keratinized tissue (KT) for the longterm maintenance and stability of peri-implant soft and hard tissues is controversially discussed..$^{1-4}$ Previous studies suggested that an insufficient KT width of $<2 \mathrm{~mm}$ can compromise peri-implant tissue stability and health over time. ${ }^{5,6}$ In particular, it was reported that areas with insufficient KT were prone to plaque accumulation, soft tissue inflammation, mucosal recession, and marginal bone loss (MBL). ${ }^{7-10}$

Several soft tissue augmentation procedures have been developed to increase peri-implant KT width and prevent biological complications around dental implants. ${ }^{4,11}$ Currently, the autogenous free gingival graft (FGG) is considered the clinical standard to gain KT width and thickness around dental implants, presenting favorable outcomes over time. ${ }^{12-14}$ Nevertheless, FGG undergoes inevitable dimensional changes during the healing phase which may affect the indications and predictability of this procedure. ${ }^{14,15}$ Currently, the majority of studies have evaluated the associated dimensional changes around teeth, reporting that FGG undergoes a shrinkage rate between $12 \%$ and $45 \%$ over a 3 - to 6 -month follow-up period. ${ }^{16-20}$ Currently, there is limited evidence evaluating the dimensional changes of FGG at implant sites. A previous experimental study in the canine evidenced that the periimplant mucosal thickness increased by $1.6 \mathrm{~mm}$ following FGG augmentation. Though, the mentioned study did not report on quantitative data to evaluate FGG dimensional changes over the 3-month observational period. ${ }^{21}$ Moreover, a retrospective clinical study evaluating the performance of FGG versus a porcine collagen matrix for KT gain at implant sites revealed that FGG augmented sites underwent a shrinkage rate of $6.65 \%, 14.59 \%$, and $28.35 \%$ after 10,30 , and 90 days of surgery. ${ }^{22}$ Similarly, two prospective clinical trials reported that FGG augmented implant sites exhibited a shrinkage rate that varied between $22 \%$ and $33 \%$ after 1 to 6 months of surgery, ${ }^{15,23}$ showing the highest dimensional alterations over the first 90 days of healing. Nevertheless, the aforementioned studies evaluated mucosal healing changes in clinical one-dimensional assessments, which in turn do not consider the complex three-dimensional changes that may occur during wound healing. Since current data are scarce to determine the real dimensional changes at FGG augmented sites, the present prospective study aimed at assessing FGG three-dimensional changes at implant sites over a 3-month follow-up period.

\section{2 | MATERIALS AND METHODS}

\subsection{Study design and participants}

For the present prospective analysis, a total of 12 partially edentulous patients (mean age: 60 years; range: 29 to 86 years), exhibiting deficient $\mathrm{KT}$ width (i.e., $<2 \mathrm{~mm}$ ) at the vestibular aspect of 19 implant sites were included. The KT width was measured with a periodontal probe ${ }^{*}$ from the zenith of the alveolar ridge to the mucogingival junction by selecting 3 measuring points (i.e., mesial, medial, and distal) at the vestibular aspect of each implant. All patients had attended the Department of Oral Surgery, Goethe University, Frankfurt-Germany for dental implant surgery (submerged healing protocol) between 2018 and 2019 and were subjected to FGG procedure at second-stage surgery at 3 to 5 months following implant placement.

The study protocol No.92/19 was approved by the human subject ethics board of Goethe-University, FrankfurtGermany and was conducted in accordance with the Helsinki Declaration of 1975, as revised in 2013. Each patient was given detailed information of the study protocol and was required to sign an informed consent form that authorized the collection of personal data and performance of digital and clinical evaluations.

This study considered the checklist items of the Strengthening the Reporting of Observational studies in Epidemiology (STROBE) statement.

\section{2 | Inclusion criteria}

The inclusion criteria considered patients who signed and approved the consent form. Patients had to meet the following criteria: 1) minimum age of 18 years old, 2) edentulous or partially edentulous participants that had

* PCV12PT Hu-Friedy, Chicago, IL 


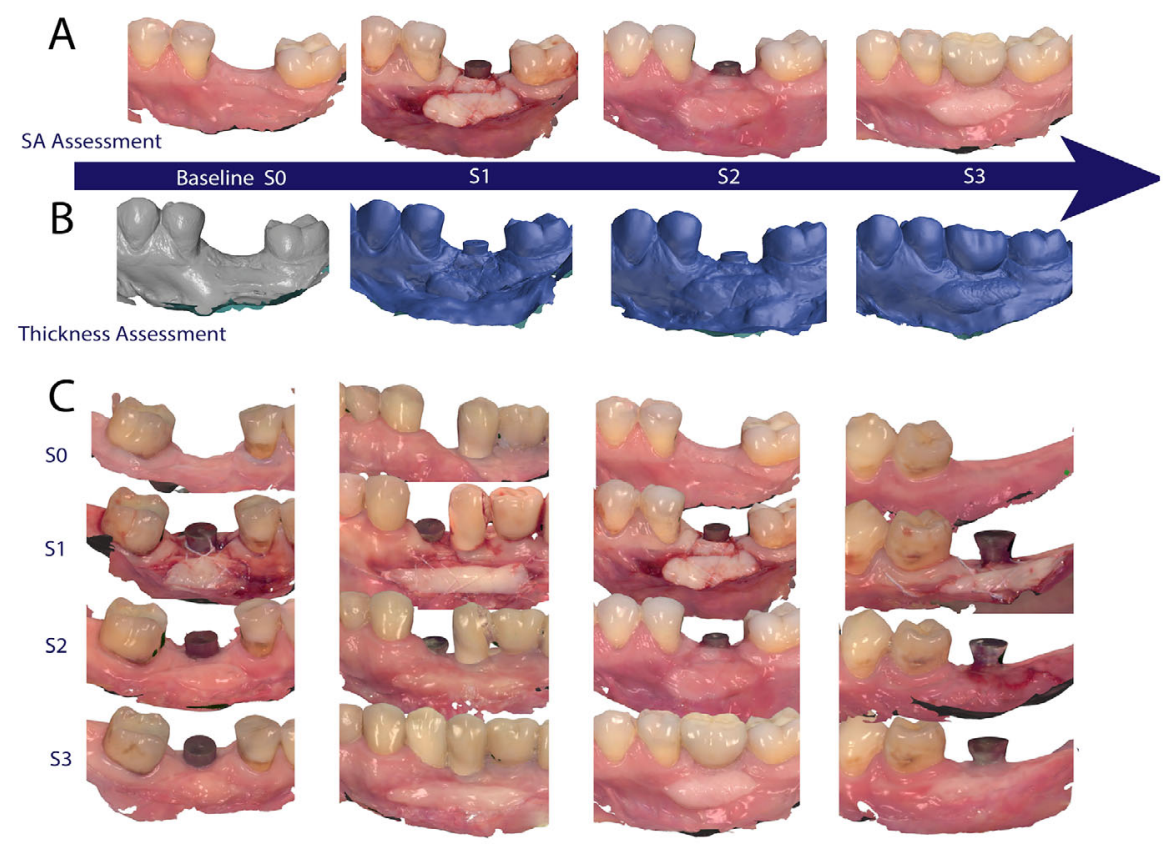

F I G U R E 1 Schematic diagram elucidating the stages of the study to evaluate SA and thickness changes over a 3-month follow-up period. The A) scan and B) STL files depict the study follow-up visits for the three-dimensional assessments. The ROI encompassing the recipient area is displayed before surgery (S0), immediately after surgery (S1), and at subsequent examination points of 30 (S2) and 90 (S3) days after surgery. C) Representative cases depicting the clinical aspect and healing process at the recipient site over the follow-up period

undergone dental implant surgery (submerged healing protocol) at grafted and/or non-grafted (i.e., pristine) sites, 3) participants exhibiting deficient KT width (i.e., $<2 \mathrm{~mm}$ ) at the vestibular aspect of the implant site and indicated for soft tissue augmentation using FGG procedure, 4) nonsmokers and/or light smokers $(<10 \mathrm{cig} / \mathrm{d}), 5)$ adequate oral hygiene as evidenced by plaque index $(\mathrm{PI})<1,{ }^{24}$ and 6) unloaded implants.

\section{3 | Exclusion criteria}

The exclusion criteria considered patients who presented: 1) general contraindications for dental and surgical treatments, 2) uncontrolled diabetes, 3) autoimmune or/and inflammatory diseases of the oral cavity, 4) active periodontal disease, 5) pregnant or lactant women, 6) patients with implants that presented complications (i.e., infection, bone dehiscence, incorrect position), that required additional treatments 7) patients who did not comply with at least 3 months of follow-up after FGG procedure, 8) patients not having complete anamnesis, clinical, and scan records, and 9) implants in function.

\section{4 | Outcome assessments}

The primary end point was defined as FGG dimensional changes subdivided in SA $\left(\mathrm{mm}^{2}\right)$ and thickness $(\mathrm{mm})$ changes over a 3-month follow-up period. FGG SA and thickness were assessed immediately after surgery (S1), as well as at 30 (S2) and 90 (S3) days after the surgical procedure (Figures $1 \mathrm{~A}$ and $1 \mathrm{~B}$ ).

Secondary end points considered FGG SA and thickness shrinkage rates (\%), wound healing (wound infections (yes/no), soft tissue dehiscence (yes/no), graft failure (yes/no) (these outcomes were recorded at day 10 as well as at 30 and 90 days after surgery), and the correlation between the initial KT width and FGG SA and the FGG shrinkage rates (SA and thickness) after 3 months of the grafting procedure. Also, the correlation between the receptor site features (jaw, region, and graft procedures) and the FGG shrinkage rate (S3) was evaluated.

\section{5 | Treatment procedures}

All FGG interventions were performed by a single experienced clinician (PP). The surgery was executed under local anesthesia (4\% articaine, 1:200,000 epinephrine). After anesthesia infiltration, the required autologous graft dimensions were outlined in the palatal mucosa with a 15stainless steel blade. The selected donor area was located between the first premolar and the first molar and at 2-mm distance from the gingival margins of the mentioned teeth. A 1-mm thickness graft was harvested from the hard palate. If present, the adipose tissue was removed from the graft's 
A

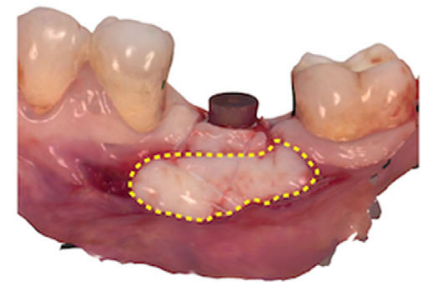

S1

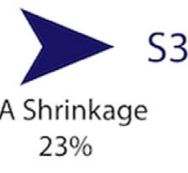

B

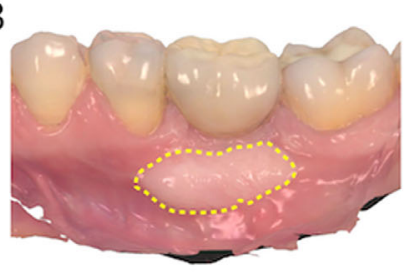

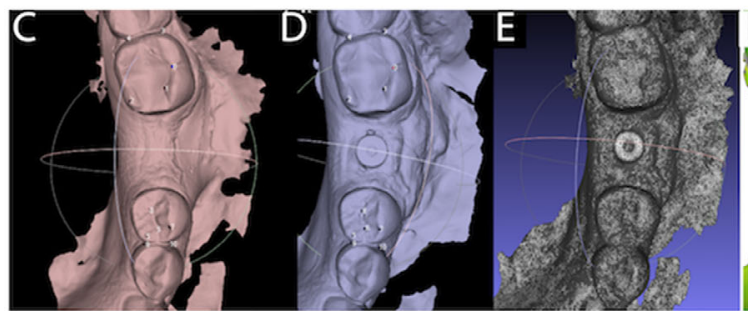
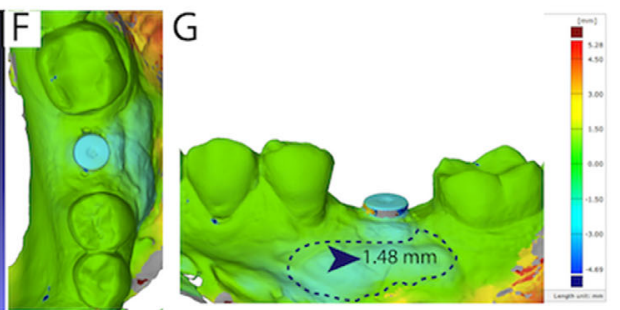

F I G U R E 2 Illustrative scan files depicting A) the delineated FGG perimeter immediately after surgery (S1) and at B) 90 days of follow-up (S3) for SA calculation. The FGG SA shrinkage rate between the mentioned periods is portrayed on the diagram. For thickness change analysis, C) the preoperative (S0) and D) postoperative (S1) STL files of the aforementioned case are aligned, and E) superimposed by the selection 10 reproducible anatomical points in each model. The thickness change between the mentioned time points is also exhibited in an F) occlusal and G) frontal view of the superimposed files where the mean FGG thickness gain of the grafted area is indicated. The multicolor color scale bar allows the interpretation of thickness changes $(\mathrm{mm})$ at the superimposed models, where negative values represent thickness gain and positive values represent loss

inner surface and thickness was measured using caliper at three aspects (i.e., mesial, medial, and distal). In case the FGG thickness was greater, the graft was thinned with the surgical blade to attain a uniform and desired width. FGG was immersed in sterile saline until placement. The donor area was cleaned with sterile saline and sutured with a $4 / 0$ non-resorbable PTFE monofilament ${ }^{\dagger}$ material to retain the clot.

The recipient bed was prepared using a 15-stainless steel blade; a horizontal split-thickness incision was performed at the mucogingival junction on the buccal aspect of the implant. In absence of KT at the receptor area, the entire mucosa at the buccal aspect of the implant was raised. The mucosa was carefully sutured to the apical region of the periosteum with 4/0 non-resorbable PTFE monofilament ${ }^{* *}$ material. The previously placed implants were uncovered and healing abutments of appropriate dimensions were inserted. The FGG was sutured and fixed to the periosteum of the recipient bed with interrupted and mattress sutures using a 4/0 non-resorbable PTFE monofilament ${ }^{* *}$ material. A periodontal dressing material ${ }^{\ddagger}$ was applied over the grafted area.

After 10 days of surgery, the periodontal dressing material and sutures were removed and an additional rinsing with $0.12 \%$ digluconate chlorhexidine was performed as anti-infective therapy. After 4 and 12 weeks of surgery, a postoperative follow-up was done to examine the heal-

\footnotetext{
${ }^{\dagger}$ Cytoplast, Osteogenics Biomedical, Lubbock, TX

${ }^{\ddagger}$ COE-PAK, GC America, Alsip, IL
}

ing conditions at the donor and recipient areas. Prosthetic treatment was performed after 3 months of surgery, following the clinical indications of the research center.

\subsection{Three-dimensional analysis}

The region of interest (ROI) covering the recipient area was intraorally scanned with an optical scanner ${ }^{\S}$ before surgery (S0), immediately after augmentation (S1), after 30 (S2) and 90 days (S3) of surgery as exemplified in Figure 1. At S3 time point, grafted implant sites were scanned before implant loading and/or immediately after prosthesis placement, as long as the marginal prosthetic contour did not have any direct contact with the graft boundaries as shown in Figure 2.

Digitalized scanned files matching with S1, S2, and S3 periods were exported to an image analysis software ${ }^{* *}$ for FGG SA analysis. The FGG perimeter was delineated with a digital pen and then its enclosed area was calculated in $\mathrm{mm}^{2}$ (Figures $2 \mathrm{~A}$ and $2 \mathrm{~B}$ ). ${ }^{25}$ The perimeter was delimited by the color contrast between the FGG and the alveolar mucosa by observing the graft boundaries in detail at a magnification of $80 \%$. In case the graft perimeter was not clear enough, the contrast tool was used to elucidate the margins. The healing abutment(s) diameter at each site served as reference to set up a numerical scale at the

\footnotetext{
$\S 3$ shape, TRIOS MOVE, Dusseldorf, Germany

${ }^{* *}$ Image J software, 1.52a, Maryland
} 
digitalized file. FGG SA shrinkage rate (\%) after 30 and 90 days of surgery was calculated applying the following formula: (S1-(evaluated time point)/S1) $\times 100$.

For thickness change evaluation, scanned files were transformed to STL format (Figures $2 \mathrm{C}$ through $2 \mathrm{G}$ ). The preoperative (S0) and postoperative (S1, S2, S3) STL files were cropped, aligned, and superimposed by selecting 10 reproducible anatomical points in each model based on previously described protocols. ${ }^{25,26,27}$ Thickness change calculations between time points ( $\mathrm{S} 0$ to $\mathrm{S} 1, \mathrm{~S} 0$ to $\mathrm{S} 2$, and S0 to S3) were executed using a software program ${ }^{\dagger \dagger}$ operating the CAD comparison tool. ${ }^{25}$ The ROI for mean thickness change quantification between the superimposed files was defined by the FGG perimeter measured at S1 (Figure 2A). The thickness shrinkage rate (\%) after 30 and 90 days of surgery was also calculated applying the mentioned formula for SA shrinkage rate.

Each analysis was performed in triplicate by one calibrated examiner (MEG). Before the analysis, an intraexaminer calibration was performed to determine the consistency and reproducibility of the measurements taken by the described method. The calibration was accepted when repeated measurements $(n=10)$ presented a intraclass correlation coefficient ranging from 0.81 to 1 .

\section{7 | Statistical analysis}

The statistical analysis was performed using a commercially available software program. ${ }^{*}$ Mean values, standard deviations, medians, and confidence intervals (CIs) for primary and secondary outcomes were calculated at patient level. In patients with multiple implants, the implant site was chosen through the software tool "simple random sampling," thus, effectively including 12 implants in 12 patients $(n=12)$. Each implant site was followed-up in regard to the graft dimensional changes occurring over the healing phase following the methodology of a similar clinical study. ${ }^{22}$ Shapiro-Wilk test was used for normality, considering $P<0.05$ significant. The Friedman analysis of variance followed by post-hoc Wilcoxon signed-rank test using Bonferroni correction was used to analyze the primary outcome results and difference between intervals. Bonferroni-adjusted significance level was set at $P<0.017$. Linear regression analyses were performed to show the relationship between the initial KM width and FGG SA (S1) and the SA shrinkage as well as thickness shrinkage rate after the 90 days of surgery (S3). Multiple regression analyses were used to evaluate the influence of the receptor site features (i.e., jaw: mandible/maxilla, region: anterior/posterior, and graft/no-graft procedures) on the

\footnotetext{
† GOM Inspect, 2018, Zeiss Company, Braunshweig, Germany

\$ SPSS v. 19.0, IBM, Chicago, IL
}

TA B L E 1 Description of implant site characteristics and frequency distributions

\begin{tabular}{|c|c|c|}
\hline Site characteristic & $\begin{array}{l}\text { No. } \\
(n=12)\end{array}$ & $\begin{array}{l}\text { Percentage } \\
(\%)\end{array}$ \\
\hline \multicolumn{3}{|l|}{ Region } \\
\hline Anterior & 1 & 8 \\
\hline Posterior & 11 & 92 \\
\hline \multicolumn{3}{|l|}{ Jaw } \\
\hline Maxilla & 5 & 42 \\
\hline Mandible & 7 & 58 \\
\hline \multicolumn{3}{|l|}{ Bone grafted site } \\
\hline Yes & 9 & 75 \\
\hline No & 3 & 25 \\
\hline \multicolumn{3}{|l|}{ Presence of KT width $(<2 \mathrm{~mm})$} \\
\hline Yes $(>0 \mathrm{~mm}$ and $<2 \mathrm{~mm})$ & 4 & 33.3 \\
\hline No $(0 \mathrm{~mm})$ & 8 & 66.6 \\
\hline
\end{tabular}

graft shrinkage rates during the 3-month follow-up period; $P<0.05$ was considered statistically significant.

\section{3 | RESULTS}

The present study was based on 12 patients (eight males and four females) presenting 19 implants that underwent soft tissue augmentation using FGG for KT gain at secondstage surgery (Fig. 1C). The statistical analysis included 12 implant sites $(\mathrm{n}=12)$ in 12 patients. The mean KT width at baseline was $0.22 \mathrm{~mm}$ ( $95 \%$ CI: 0.12 to 0.32 ). A total of seven FGG procedures were executed in the mandible, while five were performed in the maxilla. The implant sites' characteristics are presented in Table 1.

Following 3 months of surgery, 11 implants were rehabilitated with metal-ceramic cemented retained crowns (91.6\%), one implant was not rehabilitated due to a patient systemic complication, and two implants supported provisional restorations before final prosthesis placement.

Mean values for FGG SA, thickness changes, and its corresponding shrinkage rates are presented in Tables 2 and 3 and Figures $3 \mathrm{~A}$ and 3B.

Mean FGG SA (patient/implant level) amounted to 91 (95\% CI, 63 to 119), 76.2 (95\% CI, 45 to 106), and 61.3 (95\% $\mathrm{CI}, 41$ to 81$) \mathrm{mm}^{2}$ at $\mathrm{S} 1, \mathrm{~S} 2$, and $\mathrm{S} 3$, respectively. The mean FGG SA shrinkage rate was $16.3 \%$ (95\% CI, 3 to 29) from S1 to S2 (1-month follow-up) and 33\% (95\% CI, 19 to 46) from $\mathrm{S} 1$ to $\mathrm{S} 3$ (3-month follow-up), exhibiting a difference of $17 \%$ between $\mathrm{S} 2$ and $\mathrm{S} 3$ time points. The SA dimensional change from S1 to S3 was statistically significant.

Mean thickness gain from baseline (S0) to S1, S2, and S3 (patient/implant level) was 1.31 (95\% CI: 1.2 to 1.4 ), 0.82 (95\% CI: 0.5 to 1.12), and 0.37 (95\% CI.0.21 to 0.5 ) $\mathrm{mm}$, correspondingly. FGG thickness shrinkage was of $38 \%$ 
TA B LE 2 Free gingival grafts surface area mean, median, SD, and $95 \% \mathrm{CI}$ values and its corresponding shrinkage rates

\begin{tabular}{|c|c|c|c|}
\hline $\begin{array}{l}\text { Dimensional } \\
\text { parameter }\end{array}$ & S1 & S2 & S3 \\
\hline $\begin{array}{l}\text { Mean FGG SA } \\
\quad\left(\mathrm{mm}^{2}\right)\end{array}$ & 91 & 76.2 & $61.3^{*}$ \\
\hline Median & 68 & 61 & 55 \\
\hline SD & 50 & 48.2 & 31 \\
\hline $95 \% \mathrm{CI}$ & (63 to 119 ) & (45 to 106 ) & (41 to 81 ) \\
\hline $\begin{array}{l}\text { Mean FGG SA } \\
\text { shrinkage }(\%)\end{array}$ & 0 & 16.3 & $33^{*}$ \\
\hline Median & n.a & 18 & 23 \\
\hline SD & n.a. & 23 & 24 \\
\hline $95 \% \mathrm{CI}$ & n.a & (3 to 29 ) & (19 to 46$)$ \\
\hline
\end{tabular}

${ }^{*} P<0.017$, significant difference compared with S1.

TA B L E 3 Free gingival grafts thickness mean, median, SD, and 95\% CI values and its corresponding shrinkage rates

\begin{tabular}{llll}
$\begin{array}{l}\text { Dimensional } \\
\text { parameter }\end{array}$ & S1 & S2 & S3 \\
$\begin{array}{l}\text { FGG thickness change } \\
\text { (mm) from baseline }\end{array}$ & 1.31 & $0.82^{*}$ & $0.37^{*}$ \\
$\quad$ (S0) & & & \\
Median & 1.2 & 0.86 & 0.31 \\
\hline SD & 0.2 & 0.47 & 0.27 \\
\hline $95 \%$ CI & $(1.2$ to 1.4$)$ & $(0.5$ to 1.12$)$ & $(0.21$ to 0.5$)$ \\
\hline $\begin{array}{l}\text { FGG thickness } \\
\text { shrinkage (\%) }\end{array}$ & 0 & $38^{*}$ & $71.8^{*}$ \\
\hline Median & n.a. & 37 & 76 \\
\hline SD & n.a. & 36 & 22 \\
\hline $95 \%$ CI & n.a. & $(17.6$ to 58$)$ & $(60$ to 84$)$ \\
\hline
\end{tabular}

${ }^{*} P<0.017$, significant difference compared with $\mathrm{S} 1$.
(95\% CI:17.6 to 58) from S1 to S2 and 71.8\% (95\% CI: 60 to 84) from S1 to S3, presenting a difference of $33.8 \%$ between S2 and S3 time points. All thickness dimensional changes from $\mathrm{S} 1$ to $\mathrm{S} 3$ were statistically significant.

Soft tissue healing was uneventful in all patients. This was evidenced by the absence of wound infections, soft tissue dehiscence, signs of inflammation and graft failure (i.e., necrosis and mobility).

The linear regression analysis revealed a significant association between initial mean KT width and thickness shrinkage rate $(P=0.02)$ and also a significant association between initial FGG SA and FGG SA shrinkage rate $(P=0.012)$ after 90 days of surgery as portrayed in Figures $3 \mathrm{C}$ through $3 \mathrm{~F}$. No other significant associations were present.

Furthermore, multiple regression analyses revealed no significant association between the receptor site features and the graft SA and thickness shrinkage, revealing $P$ values for region $(P=0.23, P=0.41)$, jaw $(P=0.59, P=0.34)$, and grafting procedures $(P=0.1, P=0.32)$, respectively, following the 3-month observational period.

\section{4 | DISCUSSION}

The present study aimed at assessing FGG threedimensional changes at implant sites over a 3-month follow-up period. Within the limitations of a single-arm prospective study, the three-dimensional analysis revealed that FGG undergoes a substantial contraction over a 3-month healing period. This was evidenced by the assessment of FGG SA and thickness changes at grafted sites exhibiting significant dimensional alterations and shrinkage rates after 30 and 90 days of surgery.
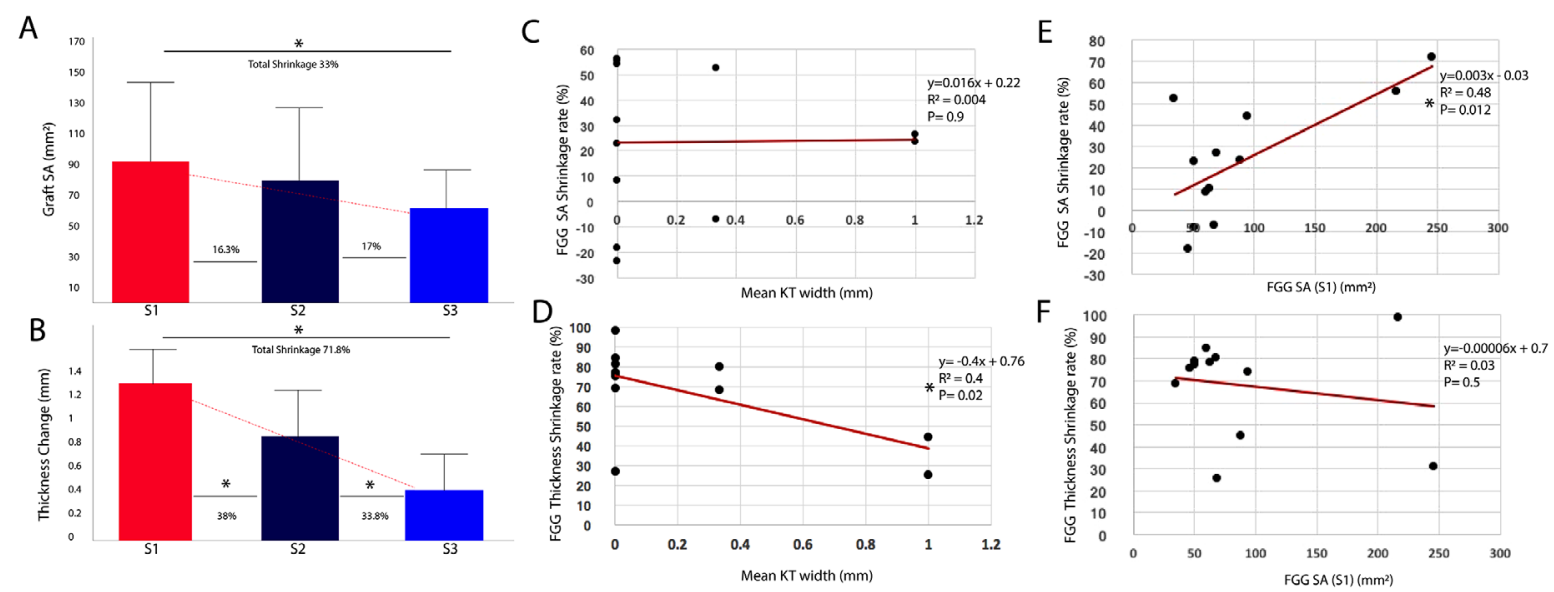

F I G U RE 3 Diagrams representing A) SA and B) thickness changes with its resultant shrinkage rates between time points, ${ }^{*} P<0.017$ considered for statistical significance. Linear regression plots to represent the relationship between mean initial KT width (mm), and C) FGG SA and D) thickness shrinkage rates and the relationship between baseline FGG SA (S1) and E) FGG SA and F) thickness shrinkage rates at 90 days of follow-up (S3). ${ }^{*} P<0.05$ considered for statistical significance 
To date, the majority of clinical studies have evaluated FGG dimensional changes around teeth, establishing that the highest shrinkage rate occurs during the first postoperative months. ${ }^{16,17,28}$ To the author's knowledge, this is the first clinical evaluation of three-dimensional (i.e., SA and thickness) changes after FGG augmentation at implant sites. Accordingly, there are limited available data which may allow for a comparison with the present results. ${ }^{29}$ A preceding experimental study in the canine assessing dimensional changes around dental implants following FGG procedure evidenced that the mucosal thickness gain after augmentation totaled to $1.6 \mathrm{~mm}$ (SD: 0.4). ${ }^{21}$ The assessment of KT thickness pointed to a similar mean value to the one noted in the present analysis immediately after FGG augmentation. However, the aforementioned preclinical study did not provide quantitative data on the thickness changes at 1 and 3 months after treatment. Furthermore, in a retrospective clinical study, Schmitt et al. ${ }^{22}$ evaluated the performance of FGG versus a porcine collagen matrix to increase peri-implant KT width in 14 patients ( $\mathrm{n}=49$ implants). FGG augmented sites underwent a shrinkage rate of $14.59 \%$ and $28.35 \%$ after 30 and 90 days of surgery. Similarly, in a randomized controlled trial of 18 patients ( $n=36$ implants), Basegmez et al. ${ }^{23}$ reported an FGG contraction rate of $28 \%$ and $33 \%$ after 90 and 180 days of follow-up, though, the contraction rate after 30 days of surgery was not evaluated. Additionally, a comparative prospective clinical trial of 21 patients ( $n=74$ implants) established that the highest FGG shrinkage rate occurred during the early healing phase, where the KT width decreased $22 \%$ and $29 \%$ after 1 and 3 months, respectively. ${ }^{15}$ Particularly, the present SA and thickness change analyses pointed to higher shrinkage rates after 90 days of surgery. Moreover, the present study exhibited greater contraction differences between 1 and 3 months of follow-up, revealing that a significant shrinkage occurred after the first postoperative month. This discrepancy could be attributed to the fact that the aforementioned studies evaluated the contraction of keratinized mucosa width in clinical one-dimensional assessments, which may not reveal the true dimensional alterations that occur at grafted areas. In contrast, the present analysis evaluated FGG two-dimensional (SA) and three-dimensional (thickness) changes which might provide a more accurate interpretation of the graft's dimensional variations over the observational period. When further interpreting volumetric changes, the present study evidenced that the thickness contraction was remarkably higher (i.e., > 70\%) compared with SA contraction (i.e., 33\%) following 3 months of augmentation. Additionally, the thickness shrinkage rate was higher (i.e., 33.8\%) after the early healing phase (i.e., second and third postoperative months) when compared with previous studies, ${ }^{15,23}$ but still lower when related to the first month of contraction (i.e., 38\%). This outcome was supported with previous data evaluating FGG changes (one- and two-dimensional assessments) at tooth and implant sites, ${ }^{15,17,20}$ indicating that the highest contraction rates happened mainly during the first postoperative month. ${ }^{15,16,18,22,30}$ It is important to consider that the discrepancy in contraction rates after the first postoperative month could be attributed to the different surgical stages, techniques (i.e., efficacy of vestibuloplasty, graft fixation, suture type) initial graft thickness, and treated regions among the studies. Moreover, the mentioned volumetric assessment and its relationship to SA contraction cannot be compared with any previous studies evaluating FGG three-dimensional changes at implant sites.

Previous studies have also suggested that FGG and recipient site features might be crucial for mucosal healing and dimensional alterations over the healing period. ${ }^{14,17}$ Consequently, the reported high contraction rates could be dependent on the surgical approach to prepare the receptor area, on the graft's size and also on the receptor site's biotype, keratinized mucosa width, previous bone grafting interventions, and areas of possible muscle pull. ${ }^{14,17,29}$ In particular, the present linear regression analyses revealed a significant association between the initial KT width (at receptor site) and the graft thickness shrinkage after 3 months of surgery, suggesting that receptor sites presenting $>0 \mathrm{~mm}$ of $\mathrm{KM}$ width $(<3 \mathrm{~mm})$ were less susceptible to thickness shrinkage over time. This finding could bring important clinical considerations to determine which cases are more prone to graft shrinkage, however the $\mathrm{R}^{2}$ value of this association was low, and this finding should be evaluated in future studies. Moreover, the multiple regression analysis did not show any significant association between the aforementioned factors (region, jaw location, and bone grafting interventions) and the graft shrinkage over the 3-month healing period. Still, future clinical studies with a larger sample size should be conducted to support the presented findings and also to assess if the receptor's site biotype at baseline could influence the graft shrinkage rate over time.

In an early clinical study, Morman et al. ${ }^{31}$ evaluated the effect of FGG thickness on the processes of shrinkage and revascularization at tooth sites and suggested that a higher shrinkage rate was expected at thinner FGGs, presenting an inverse relationship between thickness and shrinkage. Furthermore, the latter study revealed through angiographic evaluation that uniform grafts of thin to intermediate (i.e., 0.56 to $0.76 \mathrm{~mm}$ ) thickness presented a rapid vascularization when compared with thicker grafts. ${ }^{31}$ Subsequently, a possible reason for the significant thickness contraction rates observed in the present analysis could be the initial graft thickness (i.e., $1 \mathrm{~mm}$ ) that might affect shrinkage and vascularization at implant 
sites. Therefore, future studies are needed to determine the most favorable FGG thickness that will undergo the lowest contraction rates allowing a rapid vascularization at implant sites. In terms of graft uniformity, the present study included grafts presenting homogeneous thickness and comparable tissue composition since they were harvested from the same donor area among the patients. Though, the initial graft SA was different in all cases and linear regression analysis revealed a significant association between the initial FGG SA and the FGG SA shrinkage rate following 3 months of surgery, implicating that larger grafts were likely to have a higher contraction rate over the healing phase. This outcome could suggest that FGG dimensional alterations are dependent from the graft's SA at baseline, still the $\mathrm{R}^{2}$ of this association was weak, and future studies are needed to support this finding. Furthermore, a limitation of the present study is that the FGGs were placed in heterogeneous regions, exhibiting different KT widths and with the absence/presence of prior bone grafting interventions. Consequently, forthcoming clinical studies should consider the recipient site features for the inclusion criteria to avoid possible local confounders.

It is important to consider that the reproducibility of this method to analyze soft tissue changes through the superimposition of scan files needs to be assessed in upcoming clinical trials, since this is the first clinical study that applies this methodology to assess FGG dimensional changes over time. The presented outcomes are based on digital analysis which present limitations such as the possible misinterpretation of FGG boundaries when apically folded epithelial and movable scar tissues are present over the healing process. Thus, future studies should compare the presented novel methodology with other clinical/histochemical assessments such as the pulling/functional technique ${ }^{32}$ or the iodo-positive reaction $^{16,32}$ to evaluate its efficacy to identify $\mathrm{KM}$ areas. Also, histological studies ${ }^{22}$ could be performed concomitantly with the presented digital method to determine its reliability towards KM area and thickness gain evaluation. Nonetheless, all the mentioned clinical and histological methods are invasive for the patient and involved animals when compared with digital approaches, which do not physically interfere with the grafted areas.

The present study's follow-up period was based on previous pre-clinical and clinical investigations ${ }^{16,21,22,30}$ evaluating FGG dimensional changes over the first months of healing. Consistently, previous studies with longer followup periods have reported that the greatest fraction of FGG dimensional alteration occurs during the early healing phase. ${ }^{13,15,16,18,33,34}$ Still, future research should evaluate FGG three-dimensional alterations at implant sites over a longer follow-up and after different loading periods to determine if function forces could affect the graft dimen- sions over time, as the present study excluded loaded implants to avoid functional and parafunctional forces on the graft dimensional stability.

\section{5 | CONCLUSION}

Within its limitations, the present three-dimensional assessment suggests that FGGs undergo significant dimensional changes in SA and thickness over a 3-month healing period.

\section{ACKNOW LED G M EN TS}

The present study was supported by an unrestricted grant of the Osteology Foundation, Lucerne-Switzerland. The authors kindly appreciate the support provided by Dr. Karin Apaza-Bedoya who conducted part of the statistical analysis. The authors report no conflicts of interest related to this study.

Open access funding enabled and organized by Projekt DEAL.

\section{AUTHOR CONTRIBUTIONS}

Dr. Parvini contributed to conception, design, data acquisition, analysis, interpretation, and drafted and critically revised the article. Dr. Galarraga-Vinueza contributed to conception, design, data acquisition, analysis, interpretation, and drafted and critically revised the article. Dr. Obreja, Prof. Magini, and Prof. Sader contributed to data acquisition, analysis, and critically revised the article. Prof. Schwarz contributed to data analysis and interpretation and drafted and critically revised the article. All authors gave final approval and agree to be accountable for all aspects of the work.

\section{REFERENCES}

1. Berglundh T, Armitage G, Araujo MG, et al. Peri-implant diseases and conditions: consensus report of workgroup 4 of the 2017 world workshop on the classification of periodontal and peri-implant diseases and conditions. J Clin Periodontol. 2018;45:S286-S291.

2. Schwarz F, Derks J, Monje A, Wang H-L. Peri-implantitis. J Clin Periodontol. 2018;45(June 2016):S246-S266.

3. Schwarz F, Becker J, Civale S, Sahin D, Iglhaut T, Iglhaut G. Influence of the width of keratinized tissue on the development and resolution of experimental peri-implant mucositis lesions in humans. Clin Oral Implants Res. 2018;29(6):576-582.

4. Thoma DS, Naenni N, Figuero E, et al. Effects of soft tissue augmentation procedures on peri-implant health or disease: a systematic review and meta-analysis. Clin Oral Implants Res. 2018;29:32-49.

5. Boynueğri D, Nemli SK, Kasko YA. Significance of keratinized mucosa around dental implants: a prospective Comparative Study. Clin Oral Implants Res. 2013;24(8):928-933. 
6. Chung DM, Oh T-J, Shotwell JL, Misch CE, Wang H-L. Significance of keratinized mucosa in maintenance of dental implants with different surfaces. J Periodontol. 2006;77(8):1410-1420.

7. Lin G-H, Chan H-L, Wang H-L. The significance of keratinized mucosa on implant health: a systematic review; the significance of keratinized mucosa on implant health: a systematic review. $J$ Periodontol. 2013;84:1755-1767.

8. Roccuzzo M, Grasso G, Dalmasso P. Keratinized mucosa around implants in partially edentulous posterior mandible: 10-year results of a prospective comparative study. Clin Oral Implants Res. 2016;27(4):491-496.

9. Ladwein C, Schmelzeisen R, Nelson K, Fluegge TV, Fretwurst $\mathrm{T}$. Is the presence of keratinized mucosa associated with periimplant tissue health? A clinical cross-sectional analysis. Int $J$ Implant Dent. 2015;1(1):11.

10. Perussolo J, Souza AB, Matarazzo F, Oliveira RP, Araújo MG. Influence of the keratinized mucosa on the stability of periimplant tissues and brushing discomfort: a 4-year follow-up study. Clin Oral Implants Res. 2018;29(12):1177-1185.

11. Giannobile W V, Jung RE, Schwarz F. Evidence-based knowledge on the aesthetics and maintenance of peri-implant soft tissues: osteology foundation consensus report part 1-effects of soft tissue augmentation procedures on the maintenance of periimplant soft tissue health. Clin Oral Implants Res. 2018;29:7-10.

12. Oh S-L, Masri RM, Williams DA, Ji C, Romberg E. Free gingival grafts for implants exhibiting lack of keratinized mucosa: a prospective controlled randomized clinical study. J Clin Periodontol. 2017;44(2):195-203.

13. Bassetti RG, Stähli A, Bassetti MA, Sculean A. Soft tissue augmentation around osseointegrated and uncovered dental implants: a systematic review. Clin Oral Investig. 2017;21(1):53-70.

14. Zucchelli G, Tavelli L, McGuire MK, et al. Autogenous soft tissue grafting for periodontal and peri-implant plastic surgical reconstruction. J Periodontol. 2019;91(1):9-16.

15. Schmitt CM, Moest T, Lutz R, Wehrhan F, Neukam FW, Schlegel KA. Long-term outcomes after vestibuloplasty with a porcine collagen matrix (Mucograft ${ }^{\circledR}$ ) versus the free gingival graft: a comparative prospective clinical trial. Clin Oral Implants Res 2016;27(11):e125-e133.

16. Silva CO, ÉDP Ribeiro, Sallum AW, Tatakis DN. Free gingival grafts: graft shrinkage and donor-site healing in smokers and non-smokers. J Periodontol. 2010;81(5):692-701.

17. Sculean A, Gruber R, Bosshardt DD. Soft tissue wound healing around teeth and dental implants. J Clin Periodontol. 2014;41:S6S22.

18. Barbosa FI, Corrêa DS, Zenóbio EG, Costa FO, Shibli JA. Dimensional changes between free gingival grafts fixed with ethyl cyanoacrylate and silk sutures. J Int Acad Periodontol. 2009;11(2):170-176.

19. Cevallos CAR, de Resende DRB, Damante CA, et al. Free gingival graft and acellular dermal matrix for gingival augmentation: a 15-year Clinical Study. Clin Oral Investig. 2019;24(3):1197-1203.

20. de Resende DRB, Greghi SLA, Siqueira AF, Benfatti CAM, Damante CA, Ragghianti Zangrando MS. Acellular dermal matrix allograft versus free gingival graft: a histological evaluation and split-mouth randomized clinical trial. Clin Oral Investig. 2019;23(2):539-550.

21. Bengazi F, Lang NP, Caroprese M, Urbizo Velez J, Favero V, Botticelli D. Dimensional changes in soft tissues around dental implants following free gingival grafting: an experimental study in dogs. Clin Oral Implants Res. 2015;26(2):176-182.

22. Schmitt CM, Tudor C, Kiener K, et al. Vestibuloplasty: porcine collagen matrix versus free gingival graft: a Clinical and Histologic Study. J Periodontol. 2013;84(7):914-923.

23. Basegmez C, Karabuda ZC, Demirel K, Yalcin S. The comparison of acellular dermal matrix allografts with free gingival grafts in the augmentation of peri-implant attached mucosa: a randomised controlled trial. Eur J Oral Implantol. 2013;6(2):145-152.

24. Löe $H$. The gingival index, the plaque index and the retention index systems. J Periodontol. 1967;38(6):610-616.

25. Galarraga-Vinueza ME, Obreja K, Magini R, Sculean A, Sader $\mathrm{R}$, Schwarz F. Volumetric assessment of tissue changes following combined surgical therapy of peri-implantitis: A pilot study [published online ahead of print, 2020 Jun 25]. J Clin Periodontol. 2020; 10.1111/jcpe.13335.

26. Gil A, Bakhshalian N, Min S, Zadeh HH. Treatment of multiple recession defects with vestibular incision subperiosteal tunnel access (VISTA): a Retrospective Pilot Study utilizing digital analysis. J Esthet Restor Dent. 2018;30(6):572-579.

27. Schneider D, Grunder U, Ender A, Hämmerle CHF, Jung RE. Volume gain and stability of peri-implant tissue following bone and soft tissue augmentation: 1-year results from a Prospective Cohort Study. Clin Oral Implants Res. 2011;22(1):28-37.

28. Egli U, Vollmer WH, Rateitschak KH. Follow-up studies of free gingival grafts. J Clin Periodontol. 1975;2(2):98-104.

29. Thoma DS, Buranawat B, Hämmerle CHF, Held U, Jung RE. Efficacy of soft tissue augmentation around dental implants and in partially edentulous areas: a systematic review. J Clin Periodontol. 2014;41:S77-S91.

30. Ç CoşkunTürer, H Ipek, Kirtiloğlu T, Açikgöz G. Dimensional changes in free gingival grafts: scalpel versus Er:yAG laser-a preliminary study. Lasers Med Sci. 2015;30(2):543-548.

31. Mörmann W, Schaer F, Firestone AR. The relationship between success of free gingival grafts and transplant thickness: revascularization and shrinkage-a One Year Clinical Study. J Periodontol. 1981;52(2):74-80.

32. Guglielmoni P, Promsudthi A, Tatakis DN, Trombelli L. Intraand Inter-Examiner reproducibility in keratinized tissue width assessment with 3 methods for mucogingival junction determination. J Periodontol. 2001;72(2):134-139.

33. Dorfman HS, Kennedy JE, Bird WC. Longitudinal evaluation of free autogenous gingival grafts. J Clin Periodontol. 1980;7(4):316324.

34. Raterrschak KH, Egli U, Fringeli G. Recession: a 4-year longitudinal study after free gingival grafts. J Clin Periodontol. 1979;6(3):158-164.

How to cite this article: Parvini $P$, Galarraga-Vinueza ME, Obreja K, Magini RdS, Sader R, Schwarz F. A prospective study assessing three-dimensional changes of mucosal healing following soft tissue augmentation using free gingival grafts. J Periodontol. 2020;1-9. https://doi.org/10.1002/JPER.19-0640 